\title{
El poblado calcolítico "Venta del Rapa" (finales III milenio Cal. BC.), Mancha Real, Jaén. Un recinto de fosos entre las estribaciones de Sierra Mágina y el Alto Guadalquivir
}

\author{
The archaeological site of "Venta del Rapa" (late $3^{\text {rd }}$ millennium Cal. B.C) (Mancha Real, \\ province of Jaen, Spain). A pit area from the Copper Age in the Upper Guadalquivir
}

\author{
Miguel Ángel Lechuga Chica (*) \\ Marcos Soto Civantos (*) \\ M. ${ }^{a}$ Oliva Rodríguez-Ariza (*)
}

\section{RESUMEN}

Se presentan los resultados de la excavación arqueológica realizada en el poblado calcolítico "Venta del Rapa", localizado durante las prospecciones previas a la construcción de la Autovía del Olivar en el término municipal de Mancha Real, Jaén. El poblado tiene un foso en la parte más alta que delimita un área de 0,5 ha. Tanto en su interior como en su exterior se ubican cientos de estructuras excavadas en la base geológica, abarcando 2 ha. Entre ellas destacan tres tumbas colectivas de inhumación sin ajuar, cuyas dataciones radiocarbónicas, fechan su ocupación en el Calcolítico Pleno-Final (23502000 Cal. BC).

\begin{abstract}
Results are presented for the archaeological excavationa at the Copper Age settlement site "Venta del Rapa", located during the preliminary survey for the construction of the Olivar motorway within the city limits of Mancha Real, Jaén (Spain). In its highest part, this small village had a ditch which delimited a area of 0.5 ha. Outside of the ditch there were hundreds of structures, covering some 2 ha, excavated in the geological substratum. These structures included three collective burials without grave goods dated by radiocarbon to the Middle to Later Copper Age (2350-2000 Cal. BC).
\end{abstract}

(*) Instituto Universitario de Arqueología Ibérica. Edif. C-6, Universidad de Jaén. 23071 Jaén.

Correose.: mianle82@gmail.com; marcosotocivantos@gmail.com; moliva@ujaen.es

Recibido 26-IV-2013; aceptado 23-VII-2013
Palabras clave: Península Ibérica, Andalucía, Alto Guadalquivir; Calcolítico; Recinto de fosos; Ritual funerario; Dataciones radiocarbónicas, Tipología cerámica.

Key words: Iberian Peninsula, Andalucia Upper Guadalquivir; Copper Age; Pit area; Graves; Funerary ritual; Radiocarbon dating; Ceramic Typology.

\section{INTRODUCCIÓN: METODOLOGÍA Y RESULTADOS}

El Instituto Universitario de Arqueología Ibérica (Jaén) ha coordinado los trabajos de investigación arqueológica en la Autovía A 316 o del Olivar (Jaén), en el marco de las medidas correctoras propuestas para esta infraestructura. A lo largo del tramo de estudio de $35 \mathrm{~km}$ lineales se ha intervenido en 19 yacimientos, entre ellos el de "Venta del Rapa". La mayoría se vinculan claramente a la ruta de La Campiña de Jaén que discurre hacia el Valle del Guadalquivir y la Comarca de La Loma. Como el trazado de esta vía de comunicación tradicional es casi idéntico al de la actual autovía, la intervención arqueológica ha analizado una vía de comunicación histórica, vigente hasta nuestros días.

El poblado "Venta del Rapa" se localiza en el término municipal de Mancha Real, a $4 \mathrm{~km}$ al Norte del casco urbano (X 446670 Y 4186836) y en el Centro-Sur de la provincia de Jaén (Fig. 1). Se ubica sobre un pequeño cerrete, apenas destacado de su entorno, delimitado al Sur y Este por el arroyo de la Salinilla, mientras al suroeste 


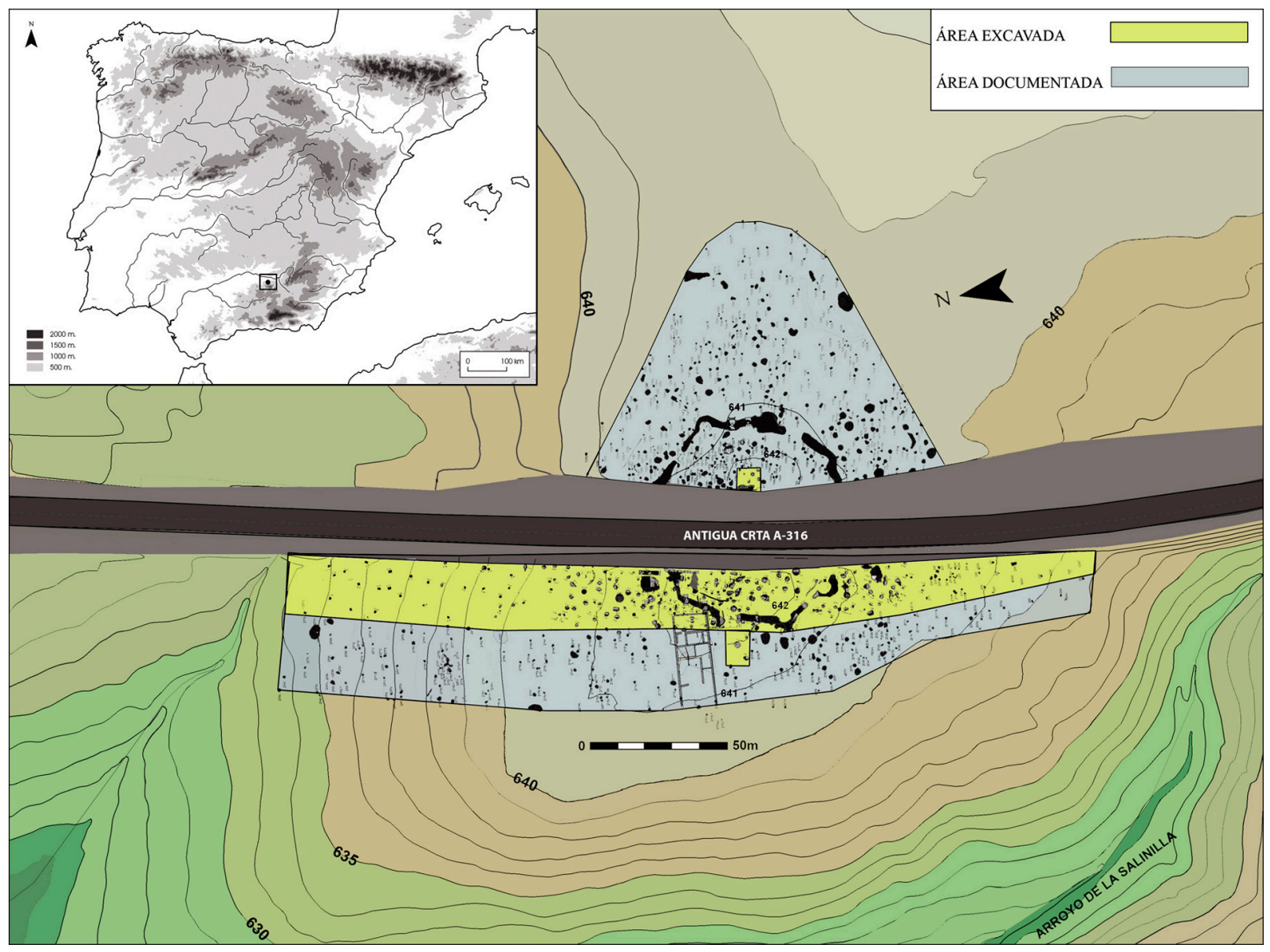

Fig. 1. Situación del poblado calcolítico "Venta del Rapa" (Mancha Real, Jaén) en la Península Ibérica; topografía y planta general del foso y las estructuras negativas domésticas y funerarias, dividida por la antigua carretera A-316.

asciende de forma casi plana, hasta la cota de los 642 m.s.n.m. El poblado se asienta sobre el límite noreste de un glacis cuaternario cuya roca del substrato es blanda, compuesta principalmente por margas. En el entorno del poblado destacan los Regosoles Calcáricos/Litosoles y Cambisoles Cálcicos, suelos normalmente con una calidad media-media baja, con un contenido medio en materia orgánica, moderadamente ácidos y una potencialidad de cultivo media-baja (1).

La ubicación del poblado no depende tanto del control visual como de su proximidad a cursos de agua, a zonas potencialmente aptas para las actividades agropecuarias, así como de la existencia

(1) López Garrido, A. C. 1971: Geología de la Zona Prebética al NE de la Provincia de Jaén. Tesis doctoral, Facultad de Ciencias, Universidad de Granada, Departamento de Geología. de un substrato geológico fácilmente excavable y la cercanía a recursos explotables como salinas. Podríamos denominar el emplazamiento 'ecotono' (Díaz del Río 2003:64), una zona de contacto entre dos ecosistemas, las estribaciones septentrionales de Sierra Mágina y la Campiña Alta de la Depresión del Guadalquivir, con amplia diversidad de recursos y donde concurren paisajes distintos.

La amplia distribución de los restos materiales en "Venta del Rapa" y la ausencia de estructuras emergentes aconsejó una doble limpieza de la superficie, previa a la excavación. Primero el cazo de una excavadora retiró la cubierta vegetal en unas 2 ha, comprendidas en los márgenes de la actuación proyectada. Después se limpiaron y delimitaron a mano las 905 estructuras detectadas, documentando y georreferenciando cada una. 
Seguidamente se procedió a la excavación del 25\% (191 estructuras en $4700 \mathrm{~m}^{2}$ ) del área que iba a ser afectada, cuya morfología era similar a un transect de más de $200 \mathrm{~m}$ de longitud y entre 20 y $25 \mathrm{~m}$ de anchura. Las 704 estructuras restantes $\left(14000 \mathrm{~m}^{2}\right)$ se protegieron mediante geotextil y quedaron soterradas bajo las infraestructuras asociadas a la autovía. La muestra excavada resulta, pues, representativa de la evolu- ción espacial de la ocupación del poblado (Fig. 1).

Primero se excavó una mitad del depósito hasta la base geológica para leer y dibujar los perfiles estratigráficos (Fig. 2) (Harris 1991) y después, en algunas estructuras, la otra mitad. Las características especiales del foso o zanja delimitado, requirieron la realización de 13 sondeos para documentar su sección, estratigrafía y accesos (Fig. 3).

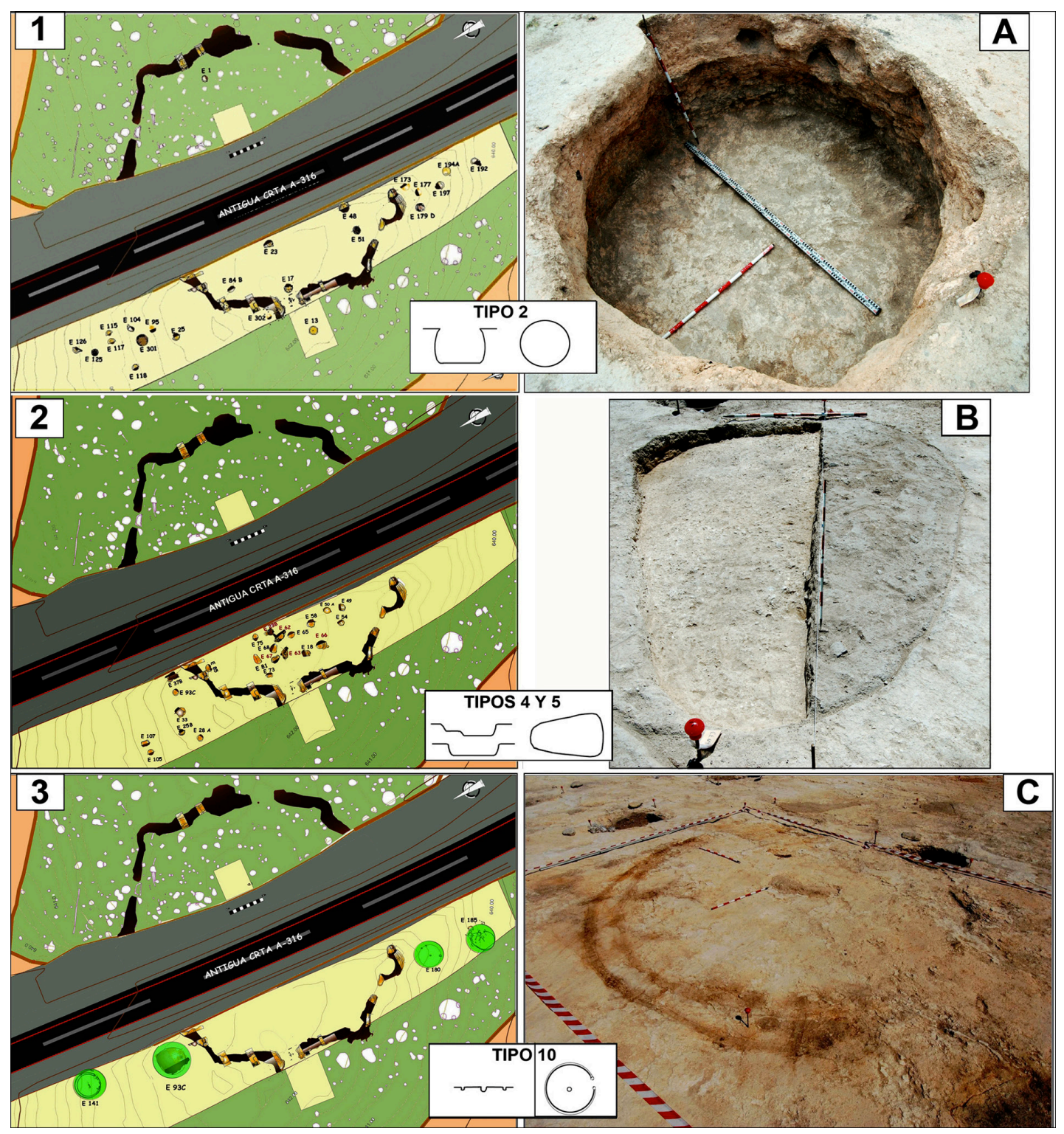

Fig. 2. Planta general del poblado calcolítico "Venta del Rapa" (Mancha Real, Jaén), situando los tipos de Estructuras de Hábitat respecto al foso (Tipo 1) y presentando su morfología y perfiles: 1. Distribución espacial de las Estructuras de Hábitat Tipo 2 en A. Fotografía planta final E.13 incluida en el Tipo 2; 2. Distribución espacial de las Estructuras de Hábitat Tipo 3 y 4, en B. Fotografía planta final E.68 incluida en el Tipo 4; 3. Distribución espacial de las Estructuras de Hábitat Tipo 4, en C. Fotografía con la delimitación inicial de E.141 incluida en el Tipo 2. 


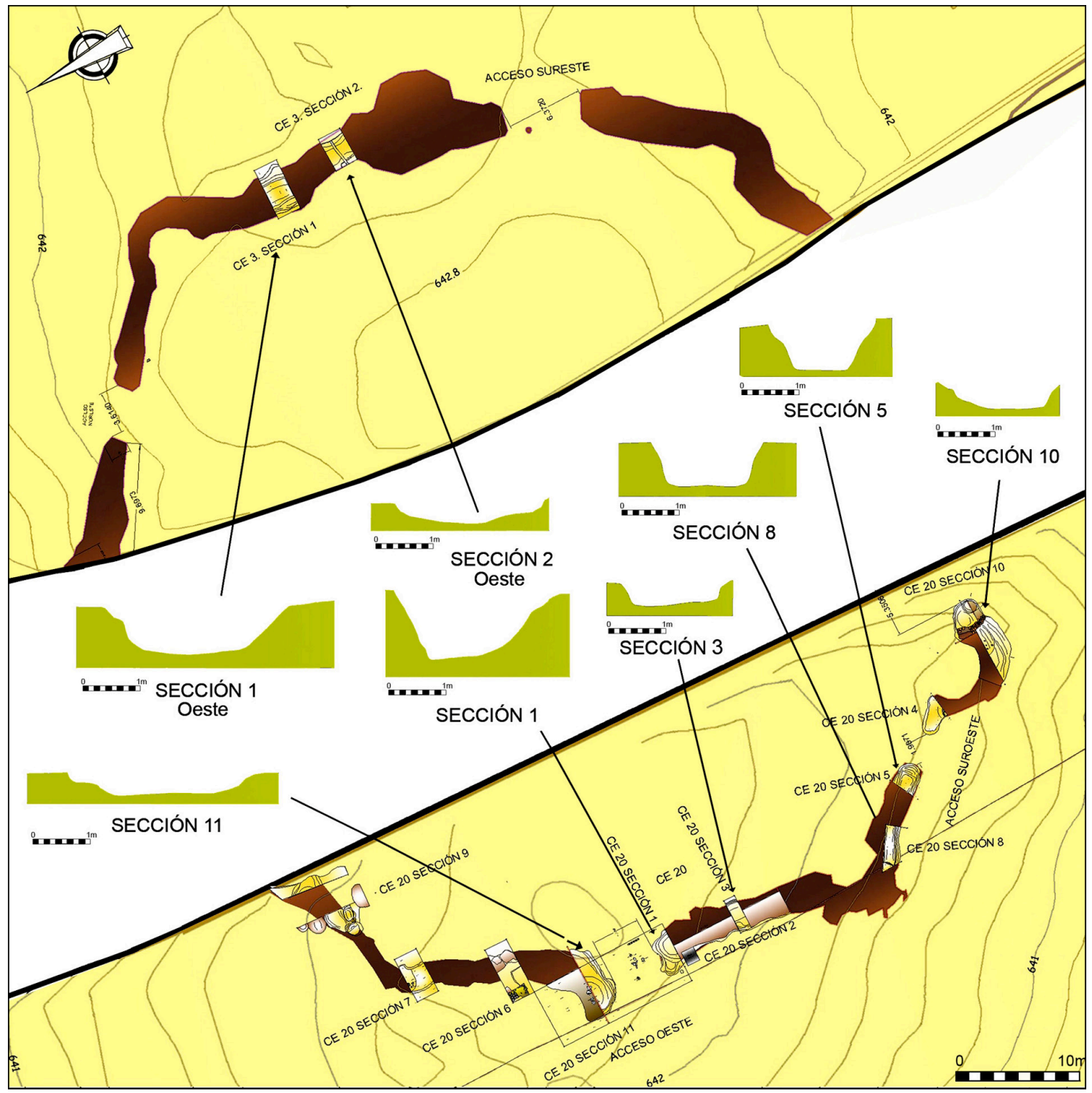

Fig. 3. Planta general del foso del poblado calcolítico "Venta del Rapa" (Mancha Real, Jaén) con la localización de las secciones realizadas.

\section{LAS ESTRUCTURAS}

Las características especiales de este tipo de poblados prehistóricos, cuya estratigrafía apenas es visible en superficie, hacen que las estructuras subterráneas sean prácticamente los únicos restos constructivos para la definición espacial de la ocupación (Lizcano 1991: 73). Resulta muy difícil establecer la secuencia de estructuras construidas durante un mismo momento de ocupación, en general sin conexión estratigráfica directa, y don- de el depósito arqueológico solo se conserva en el interior de las mismas. Tampoco podemos establecer una relación directa de coetaneidad entre las estructuras localizadas al interior y exterior del foso ante la ausencia de relaciones estratigráficas. Sin embargo podemos reconocer agrupaciones espaciales de estructuras definidas por criterios morfológicos, basados en parámetros cuantificables (dimensiones, superficie de la base, potencia conservada, anchura de la boca y de la base, situación respecto al foso), así como deter- 
minar su funcionalidad cuando el estudio estratigráfico y de materiales lo permitía.

Se definieron un total de 10 tipos atendiendo a criterios morfológicos y constructivos, rara vez matizados por criterios funcionales, dada la ausencia generalizada de contextos deposicionales. El Tipo 1 es el foso. Asignamos alrededor del $20 \%$ del total de estructuras excavadas, a los Tipos 2, 4, 5, 10, interpretados como espacios de hábitat o cabañas. Los Tipos 3, 6, 7, 8 y 9 se asociarían con otros usos (almacenaje, molienda, despiece, hogares), en su mayoría difíciles de determinar.

\subsection{Las Estructuras de hábitat. Tipos 2, 4, 5 y 10}

El Tipo 2 comprende 23 estructuras. Son cabañas de morfología circular con diámetro de la base siempre mayor que el de la boca y sección acampanada más o menos acentuada. El diámetro medio de la base es de unos $2 \mathrm{~m}$ y el de la boca ronda el 1,6 $\mathrm{m}$. La profundidad media conservada es de 1,3 $\mathrm{m}$. La base es plana y de una superficie media de unos $3,8 \mathrm{~m}^{2}$. Se abren principalmente al exterior del foso, en dos núcleos situados en las laderas septentrional y meridional, lo que sugiere que el foso no fue un factor decisivo de localización (Fig. 2:1A).

Los Tipos 4 y 5 (fondos de cabaña, estructuras emergentes), muy parecidos morfológicamente, tienen ciertas diferencias. En el Tipo 4 incluimos 5 estructuras excavadas en parte en la base geológica. Su potencia oscila entre los 40 y los 90 $\mathrm{cm}$ y la superficie media de la base en torno a los $4 \mathrm{~m}^{2}$. Tienen una morfología irregular de tendencia ovalada con los extremos redondeados y, en su mayoría, sección en ' $U$ '. La base suele estar escalonada, siendo mayor el escalón más profundo. La presencia habitual de hoyos de poste en torno a la boca, sugiere su empleo para sustentar una cubierta de entramado vegetal. Este tipo de estructuras se localiza dentro del foso en su zona occidental, concentrándose en unos $150 \mathrm{~m}^{2}$. Al Tipo 5 se adscriben 15 estructuras de morfología irregular y clara tendencia ovalada. La superficie de la base ronda los 3,3 $\mathrm{m}^{2}$. La potencia conservada suele estar entorno a los 30-40 cm. Presentan sección en ' $U$ '. Dentro del foso aparecen 10 (el $62,5 \%)$ y las 5 restantes en la ladera septentrional a no más de $20 \mathrm{~m}$ del mismo (Fig. 2:2B).
Suponemos que tenían alzado y cubierta formados por un entramado vegetal, del que no se han conservado restos, ni marcas en la base geológica.

Hemos asignado al Tipo 10 solo 4 estructuras emergentes de surco perimetral. Los restos conservados escasean al haberse construido las cabañas en superficie con materiales perecederos salvo los hoyos de poste y el surco de 'cimentación'. Este delimita un espacio circular, entre 5 y $6,8 \mathrm{~m}$ de diámetro, cuya superficie interior oscila entre los 20 y los $37 \mathrm{~m}^{2}$. En su trazado hay algún hoyo de poste circular entre 10 y $20 \mathrm{~cm}$ de diámetro que reforzaría el alzado de la estructura. El acceso al interior está marcado por un espacio sin surco de 0,5-1,1 $\mathrm{m}$ de anchura, delimitado por dos hoyos de poste. Este acceso se suele situar en el límite oriental o sureste (Fig. 2:3C). Al menos dos de ellas se reconstruyen o estructuran de nuevo, lo que atribuimos a la fragilidad de su construcción que requeriría sucesivas ocupaciones y reforzamientos. Los alzados de estas estructuras no se conocen. En poblados cercanos de la misma época como Marroquíes Bajos se ha propuesto que consistieran en un cuerpo bajo cilíndrico y cubierta cónica, formada por un entramado de madera y ramajes flexibles, reforzado con algunos postes en el perímetro y la entrada y con un posible cubrimiento de barro para mejorar el aislamiento. Uno o varios postes centrales soportarían el peso de la cubierta (Zafra de la Torre et al. 1999:84; Serrano et al. 2011: 125-126). La concentración de las 4 estructuras fuera del foso (Fig. 2:3) puede estar determinada por su pésima conservación. Numerosos hoyos de poste (Tipo 9), distribuidos dentro y fuera del foso, podrían formar parte de cabañas similares cuyos restantes elementos han desaparecido.

\subsection{El foso o zanja. Tipo 1}

El trazado de la antigua carretera A-316 dividió el poblado y el foso en una Zona Este y otra Oeste. En cada zona abrimos en el foso 2 y 11 secciones respectivamente (Fig. 3). El foso o zanja está excavada en la base geológica, ubicada en la parte más alta del poblado. De morfología circular ligeramente irregular y tendencia hexagonal mide unos $70 \mathrm{~m}$ de diámetro. Su perímetro de algo más de $235 \mathrm{~m}$ está segmentado por los accesos al recinto y abarca una superficie de 4450 
$\mathrm{m}^{2}$. Su anchura varía entre 1 y $3 \mathrm{~m}$. Tiene sección en ' $U$ ' y una potencia entre 0,3 y $1,6 \mathrm{~m}$. El foso estaba colmatado por niveles postdeposicionales naturales y vertidos de posible origen antrópico. La presencia de adobes en varios niveles y en el trazado superficial del foso apunta a su empleo en la construcción de un pequeño murete o reborde interior en la mayor parte del recorrido. Los 5 accesos al interior del recinto tienen entre 3 y 6 $\mathrm{m}$ de anchura. Se sitúan a distancias regulares sin excluir que hubiera al menos otro destruido por los antiguos desmontes de la Carretera A-316. La planta general (Fig. 3) muestra una serie de 'salientes' o pequeños bastiones de tendencia semicircular con diámetro entorno a los $2 \mathrm{~m}$. Situados a 10-12 m de los diferentes accesos, remarcan los giros que dan al foso su característica tendencia circular.

La funcionalidad del foso tendría un matiz ideológico y cultural más que defensivo, ya que sus dimensiones no sugieren que fuera un elemento infranqueable. Descartada también una función hidráulica, pudo actuar como barrera efectiva y simbólica probablemente delimitando el espacio del grupo. Además su funcionalidad debió estar estrechamente relacionada con el importante papel que la ganadería jugaba en la pequeña comunidad que lo habitaba, actuando como 'barrera o cerca' donde resguardar la cabaña ganadera. Su construcción requirió un esfuerzo comunal mediante un proceso de agregación de varios grupos familiares. El foso actuaría así como medio de afirmación de la cohesión interior de la comunidad.

Entendemos el foso de "Venta del Rapa" como elemento vertebrador de la distribución espacial de las estructuras contemporáneas a su creación y uso hasta que, en un determinado momento de la ocupación, perdió su función original. Entonces, se permitió y favoreció su colmatación e, incluso una vez concluida, se construyeron algunas estructuras sobre su trazado, como ocurre en el Foso 0 del citado Marroquíes Bajos de dimensiones y morfología parecidas pero de cronología más temprana (Rodríguez-Ariza et al. 2005: 591, 2006: 289).

\subsection{Otras estructuras}

Nos ha sido complicado asignar un uso a las estructuras agrupadas en los Tipos 3, 6, 7, 8 y 9.
Las del Tipo 3 corresponden a almacenes o silos de sección acampanada. Solo 4 de las 24 estructuras incluidas en esta categoría se localizan en el foso. En cambio la funcionalidad de los Tipos $6,7,8$ y 9 es imposible de determinar. Son pequeñas fosas de tendencia circular de menos de $1 \mathrm{~m}$ de diámetro con diferentes profundidades. Contienen un reducido número de artefactos (restos de sílex, fragmentos de molinos o almagra) en posición estratigráfica secundaria.

\section{LOS MATERIALES}

Ante la elevada presencia de material cerámico recuperado en la excavación, planteamos la necesidad de realizar el estudio específico de este tipo de material. La muestra seleccionada comprende 533 fragmentos cerámicos provenientes de los tipos definidos como estructuras de hábitat. En su amplia mayoría proceden de niveles postdeposicionales, por lo que los materiales se analizaron por estructuras, con independencia de su origen estratigráfico.

Pocas publicaciones profundizan en el tema de la cerámica prehistórica en la provincia de Jaén. Falta una tipología clara asociada a cronologías precisas. En este trabajo, tras revisar varias (Otero 1985; Murillo et al. 1991; Cámalich y Martín 1999; Fernández 2008), se optó por tomar como base la tipología del poblado de Los Castillejos de Montefrío (Granada) (Arribas y Molina 1979), introduciendo también los criterios funcionales definidos en el estudio tipológico de la Parcela C de Marroquíes Bajos (2).

El Tipo I comprende todo tipo de piezas decoradas (incisas, impresas, pintadas, etc.). Los fragmentos son muy escasos: 8 están pintados a la almagra $(1,50 \%$ del total) y 7 incisos y con trazos impresos $(1,31 \%$ del total). Los últimos forman espigas u hojas de acacia, habitualmente en el fondo de los cuencos, o puntillados y formas geométricas incisas, mayormente triángulos rellenos de puntillado, en vasos carenados (Fig. 4). En los Tipos II (cerámicas con las superficies tratadas) y III ( $\sin$ tratamiento de las superficies) el

(2) Rodríguez-Ariza, M. O.; Luna, M. B.; Montes, E. y Visedo, A. (2006) Memoria de las excavaciones realizadas en la Parcela $C$ de Marroquies Bajos. Delegación Provincial de Cultura de Jaén. Consejería de Cultura. Junta de Andalucía. Inédito.

Trab. prehist., 71, N. ${ }^{\circ}$ 2, julio-diciembre 2014, pp. 353-367, ISSN: 0082-5638

doi: $10.3989 /$ tp.2014.12139 
TIPO I. Cerámicas con decoración. Subtipo 1. Decoración incisa. Subtipo 2. Decoración pintada

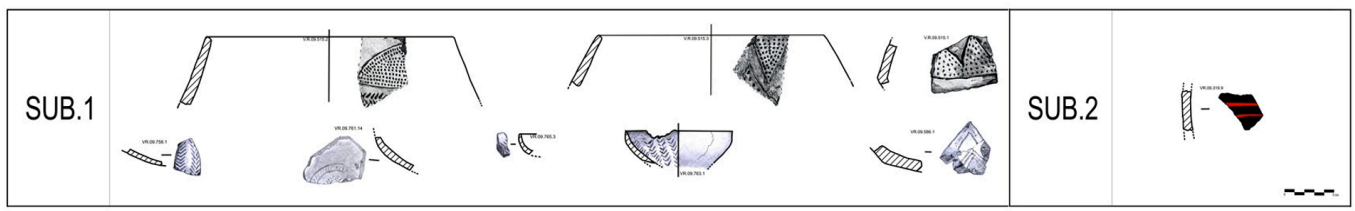

TIPO II. $\quad \begin{aligned} & \text { Formas abiertas con las superficies tratadas. } \\ & \text { 1. Cuencos. Subtipo 2. Vasos. Subtipo 3. Platos. Subtipo 4. Fuentes }\end{aligned}$

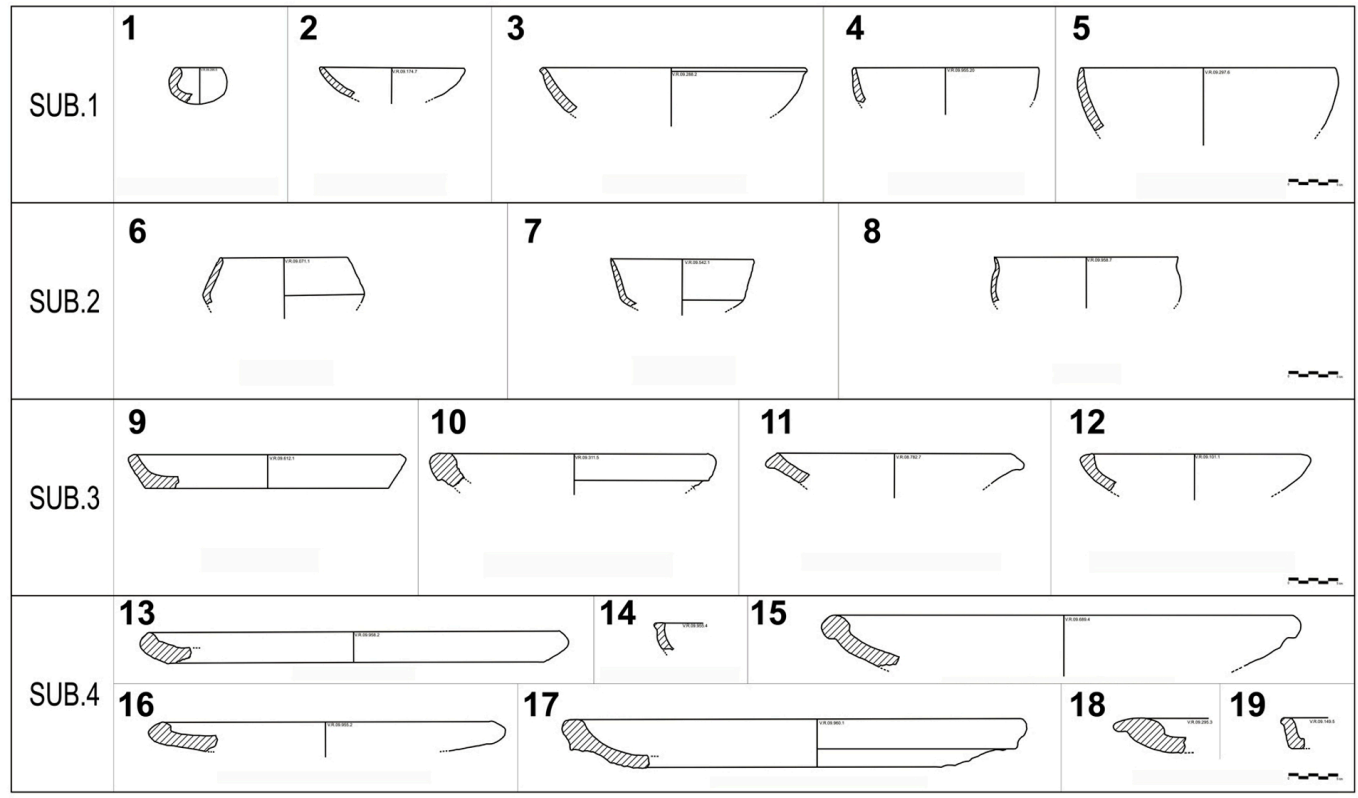

TIPO III. Cerámica lisa no cuidada. Subtipo 1. Ollas. Subtipo 2. Orzas

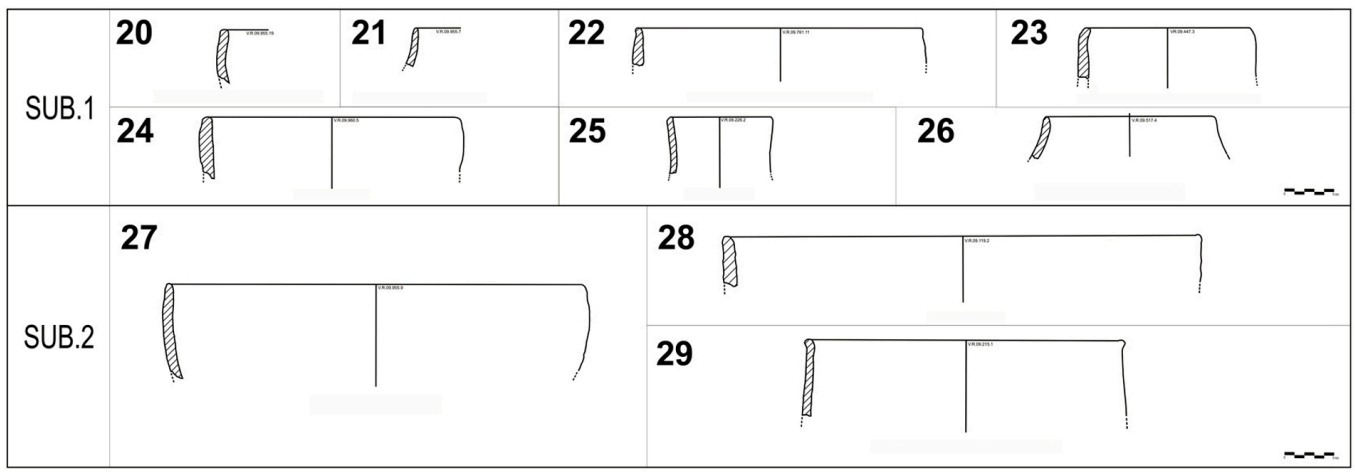

Fig. 4. Tipología de los recipientes cerámicos del poblado calcolítico "Venta del Rapa" (Mancha Real, Jaén) 1. Cuenco o vasito pequeño de paredes gruesas. 2. Cuenco plano de casquete esférico. 3. Cuenco grande de casquete esférico. 4. Cuenco de casquete semiesférico. 5. Cuenco grande de casquete semiesférico. 6. Vaso de carena media. 7. Vaso de carena baja. 8. Vaso de Perfil en "S". 9. Plato de perfil sencillo. 10. Plato de borde engrosado indicado en ambas caras. 11. Plato de borde engrosado indicado en la cara externa. 12. Plato de borde engrosado indicado en la cara interna. 13. Fuente no carenada de borde simple. 14. Fuente de borde engrosado y labio plano. 15. Fuente no carenada de borde engrosado indicado en ambas caras. 16. Fuente no carenada y borde engrosado en la cara interna. 17. Fuente no carenada y borde engrosado en la cara externa. 18. Fuente plana con borde engrosado y saliente. 19. Fuente plana con borde indicado en la cara externa. 20. Olla o cuenco de borde ligeramente entrante. 21. Olla con borde marcadamente entrante. 22. Olla con borde vertical ligeramente vuelto hacia el exterior. 24. Olla con borde saliente. 25 . Vaso con gollete. 26. Ollita de borde saliente y cuello marcado. 27. Orza de borde ligeramente entrante. 28. Orza de borde saliente. 29. Orza con borde ligeramente vuelto al exterior. 
$99,43 \%$ de los fragmentos corresponden a formas abiertas. Prácticamente la mitad son cuencos, especialmente de casquete semiesférico y esférico, acompañados por ollas y orzas de bordes saliente o ligeramente entrante, fuentes y platos de labio engrosado, vasos, etc. (Fig. 4). La mayoría de las pastas son oscuras con cocciones oxidantes y reductoras y desgrasantes de tamaño medio. Las superficies suelen estar alisadas en la cara interna en el caso de cuencos, fuentes, platos y vasos y en ocasiones en ambas. En general las fuentes y platos se hicieron con algún tipo de molde vegetal, ya que presentan improntas en la base. Las superficies bruñidas destacan entre las que cuentan con algún tratamiento. Estos bruñidos aparecen más en los cuencos que en las fuentes y platos y, en absoluto, en los vasos, ollas y orzas. Los bruñidos son más bien leves y se hacen sobre pastas negras y cocciones reductoras.

Además se crearon tres grupos funcionales distintos. El de cocina agrupa vasijas, como las ollas, destinadas a la elaboración y manipulación de alimentos. El de consumo engloba los cuencos, las fuentes, los platos y los vasos, utilizados para servir y contener los alimentos. El de almacenamiento incluye, entre otras, las orzas dedicadas a la contención y el transporte de los alimentos. Porcentualmente hay evidentes diferencias entre los grupos funcionales (Fig. 5). Destacan las piezas destinadas al consumo $(69,65 \%$ del total), sobresaliendo los cuencos $(50,09 \%$ del total) frente a las fuentes y platos $(2,75 \%)$. Les siguen las 50 piezas de cocina $(8,96 \%)$ y los

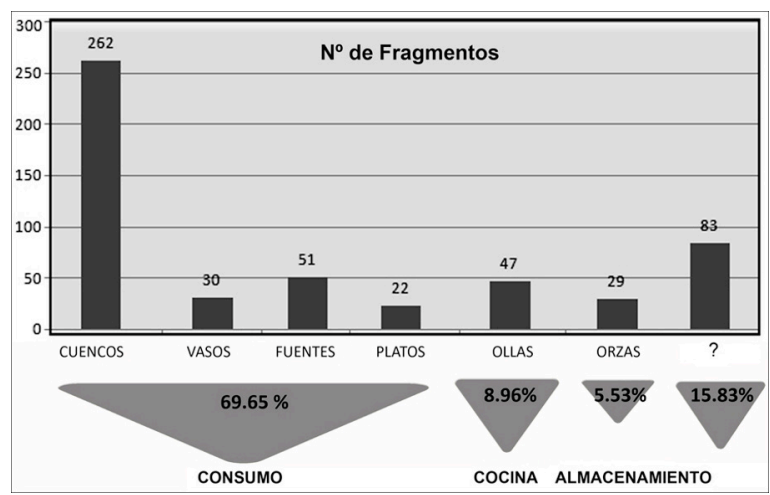

Fig. 5. Representación gráfica por número de fragmentos de los grupos cerámicos del poblado calcolítico "Venta del Rapa" (Mancha Real, Jaén), según tipología y porcentaje de los grupos funcionales definidos. Indeterminados:? contados recipientes de almacenaje (5,53\%). Los fragmentos cuyas formas no han permitido identificar claramente el uso de los ejemplares de los que proceden represetan el 15,83\%.

Además en el poblado se han documentado crecientes de arcilla cocida. Estos objetos en forma de rollo arqueado con los extremos perforados se interpretan como pesas de telar (Serrano et al. 2011: 105), indicativas de una actividad textil en el poblado. Destaca un fragmento documentado en un nivel de uso de la Estructura 13. En un nivel postdeposicional (US2A) de la mis$\mathrm{ma}$, se recuperó parte de una figurilla ginecomorfa de terracota de $5 \mathrm{~cm}$ de altura. Tiene ambos extremos (supuestos pies y cabeza) engrosados y una zona central más estilizada con pechos claramente identificables que destacan sobre la uniformidad de la pieza (Fig. 6).

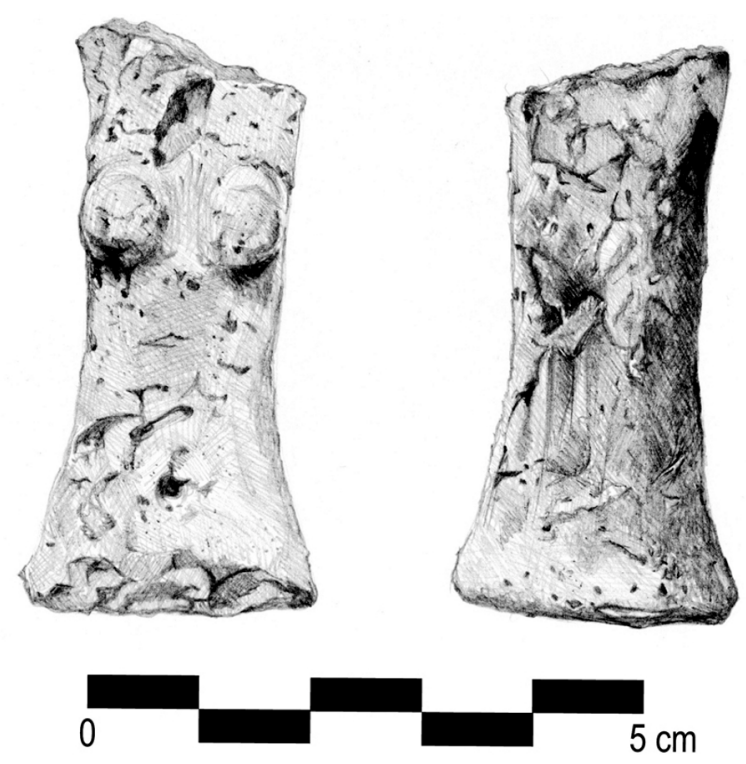

Fig. 6. Figura ginecomorfa en arcilla procedente de un nivel postdeposicional (US2A) de la Estructura 13 del poblado calcolítico "Venta del Rapa" (Mancha Real, Jaén).

El material lítico de la intervención es escaso, apenas 19 piezas. Las herramientas asociadas a tareas agrícolas son 2 azuelas y 1 hacha, todas pulimentadas $(15,78 \%)$. Hay 6 molinos (3 completos), asociados al procesado de cereal $(36,84 \%)$, 1 destinado al procesado de almagra y 1 mano de molino o machacador. Los 8 elementos $(42,10 \%)$ 
de la industria tallada sobre sílex tienen tratamiento bastante tosco y poco cuidado: 5 fragmentos de cuchillo, 1 raspador y 2 fragmentos sin forma identificada.

\section{LA NECRÓPOLIS}

Fuera del foso se documentaron 3 estructuras funerarias de morfología similar a las del Tipo 2 . Son fosas excavadas en la base geológica de sección acampanada más o menos acentuada. La Estructura Funeraria 125 se localiza a $35 \mathrm{~m}$ al Norte del foso. Presenta una morfología circular, sección ligeramente acampanada, diámetro en la base de 1,8 $\mathrm{m}$ y potencia conservada de 1,3 m. En la parte superior de las paredes dos entrantes, enfrentados, posiblemente sirvieron para sustentar algún tipo de cierre de la fosa. Su base es completamente plana. Hemos diferenciado hasta 3 estratos de inhumaciones. El primero (US6) descansa sobre la base y presenta restos escasos e indeterminados de fauna (3). Según el estudio antropológico (4) existe un Número Mínimo (NM) de 10 individuos. Solo un varón maduro está en clara conexión anatómica, en posición fetal. De los restantes solo aparecen fragmentos óseos inconexos (Fig. 7:1). En el segundo nivel de inhumaciones con un NM de 13 Individuos se reconocen 6 en conexión anatómica, también en posición fetal. Al menos aparecen dos cráneos aislados junto a las paredes de la estructura (Fig. 7:2). En el tercer nivel de inhumaciones se determina un NM de 13 individuos. Su estado de conservación es más deficiente, pero en casi la mitad de ellos se intuye una conexión anatómica en posición fetal (Fig. 7:3). En suma, en la E125 se ha determinado un NM de 36 individuos. Algunos individuos presentan conexión anatómica, pero la mayoría no, posiblemente por el uso continuado de la estructura y por su funcionalidad como osario al que solo se trasladarían algunos restos de los fallecidos. Destacamos la ausencia

(3) J. A. Riquelme Cantal (Dpto. Prehistoria y Arqueología. Universidad de Granada) realizó el estudio arqueozoológico (inédito) (2010). Archivo de la Delegación Provincial de Cultura de Jaén. Consejería de Cultura. Junta de Andalucía.

(4) J. Sebastián Martín Flórez y Zita Laffranchi (Laboratorio de Antropología Física, Universidad de Granada) elaboraron el estudio paleoantropológico (inédito) (2010). Archivo de la Delegación Provincial de Cultura de Jaén. Consejería de Cultura. Junta de Andalucía. de elementos de ajuar, asociados al nivel de inhumaciones, bien porque no los poseyeran, bien porque fueran artefactos/ecofactos que no se han conservado.

La Estructura Funeraria 179D se localiza en la vertiente meridional, a escasos $5 \mathrm{~m}$ del foso. Presenta una morfología circular, diámetro en la base de $2,1 \mathrm{~m}$, potencia conservada de $1,6 \mathrm{~m}$, sección acampanada y fondo plano. En la parte alta de las paredes hay diferentes marcas de postes posiblemente utilizadas para sustentar algún tipo de cierre o cubierta. Sobre la base se suceden un pequeño nivel de tierra (US6), una capa homogénea de piedras y otra de sedimento sobre la que se realizan 6 inhumaciones y se deposita un cánido. El estado de conservación de los individuos no es óptimo pero permite intuir la conexión anatómica de los restos en posición fetal con los cráneos junto a las paredes de la estructura. Es destacable la ausencia de cualquier tipo de ajuar. Los restos óseos aparecen cubiertos por una capa de la base geológica descompuesta.

La E301 se ubica a escasos $20 \mathrm{~m}$ al Norte del foso. La estructura es de morfología circular, sección acampanada y fondo plano, con un diámetro en la base de $2,5 \mathrm{~m}$ y $1,9 \mathrm{~m}$ en la boca y una potencia conservada de 1,4 m. Esta estructura presenta un primer nivel de habitación asociado al hábitat (US8) sobre el que se documenta una capa de piedras, principalmente en torno a las paredes pero sin distribución homogénea. Sobre ella se depositan 19 individuos inhumados, así como restos de, al menos, dos perros. Los individuos se disponen en posición fetal, normalmente con el cráneo junto a las paredes. Sobre las inhumaciones se documenta un nivel de piedras 'sellando' la estructura. Tampoco en esta tumba hay elementos de ajuar asociados al nivel de inhumaciones.

El estudio antropológico ha determinado 61 individuos en las tres tumbas (Tab. 1). Entre los adultos mayores de 21 años hay 20 mujeres, 21 hombres y 2 alofisos. Se han determinado 18 individuos no adultos: 12 son Infantil II, 5 Infantil I y 1 Infantil sin especificar. La esperanza de vida de esta comunidad se situaría entre los 25 y los 35 años, con un elevado índice de mortalidad en las edades más tempranas. Solo un reducido número de sus miembros alcanzaría más de los 40 años. La distribución por sexo es casi paritaria en conjunto, pero no por tumbas. En la E125 aparecen 17 hombres, 7 mujeres y 12 niños; en 


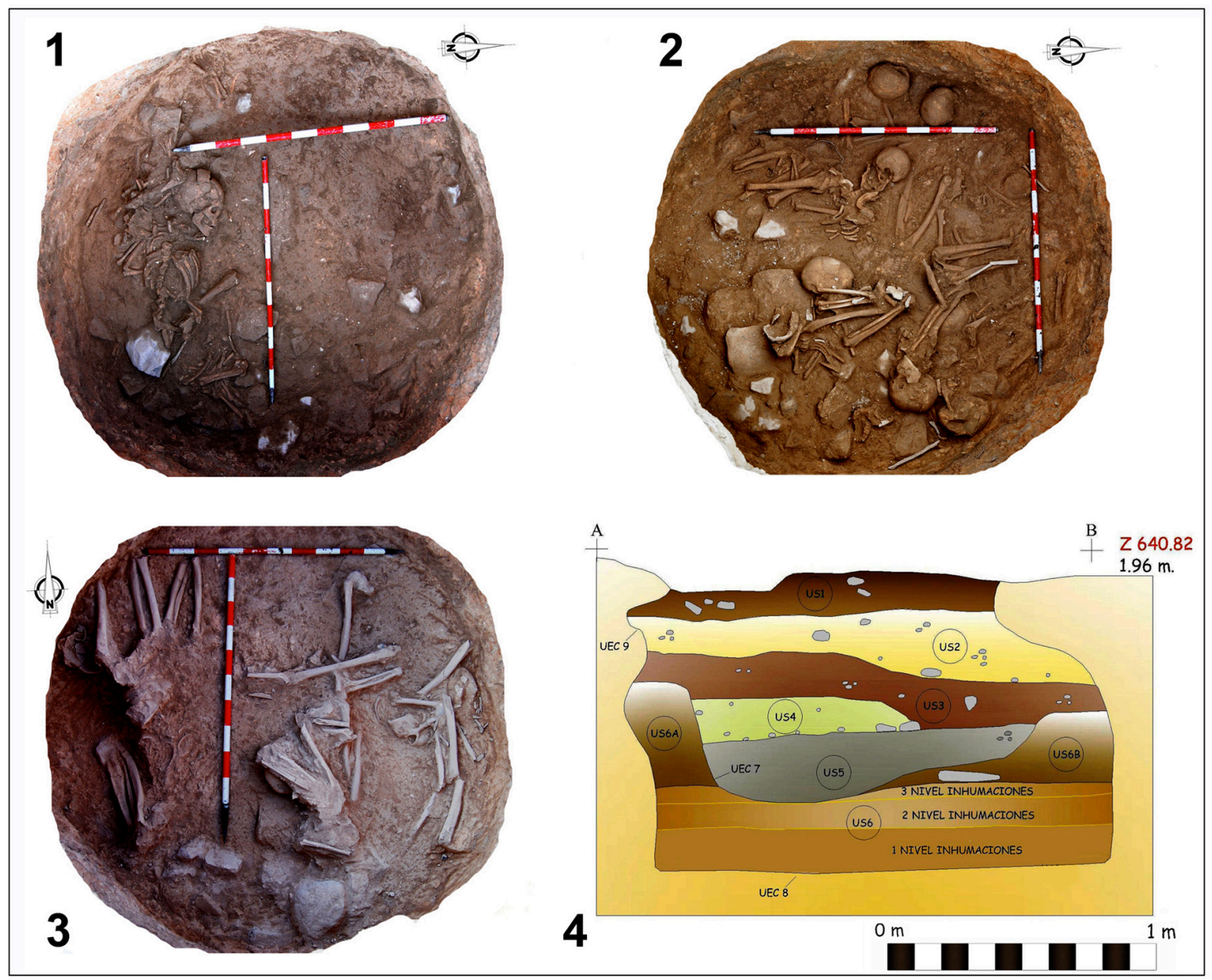

Fig. 7. Sepultura E.125 del poblado calcolítico "Venta del Rapa" (Mancha Real, Jaén) con los tres niveles de inhumaciones y el perfil estratigráfico documentado.

la E301, a la inversa, 9 mujeres, 2 hombres y 8 niños y, por último, en la E179D 4 mujeres y 2 hombres (Fig. 8).

En conclusión, gran parte de los miembros de la comunidad eran inhumados en tumbas colectivas, carentes de ajuar, sin diferencias entre individuos y acompañados por perros en dos de las tres tumbas, un elemento 'ritual' común en el Alto Guadalquivir (Lizcano 1993: 112). El enterramiento múltiple, característico del Calcolítico de la zona, es interpretado comúnmente como resultado de la preponderancia del sentimiento de pertenencia al grupo (Lomba et al. 2009:156). En la muestra analizada ambos sexos y todos los segmentos de edad están representados en amplias proporciones, lo que parece indicar que es- tamos ante la inhumación de gran parte de la comunidad y no de una selección de la misma. Sin embargo, la diferente distribución de sexos en dos de las tumbas nos plantea alternativas como que responda a una composición aleatoria de los grupos familiares enterrados o a una agrupación en base al sexo o a un acceso diferencial al enterramiento colectivo. Estas cuestiones que se han planteado en yacimientos cercanos como el Polideportivo de Martos (Cámara 2001) o más alejados como el del Camino de las Yeseras en la provincia de Madrid (Blasco y Ríos 2012) y esperamos poder ir definiéndolas en un futuro próximo.

La presencia de cánidos, relacionada con la ganadería (Cámara 2001: 66), puede estar reco- 


\begin{tabular}{|c|c|c|c|}
\hline RANGOS DE EDAD & E. 125 & E. 301 & E. 179 D \\
\hline VARONES MADUROS (41 - 60 años) & 1 & 0 & 0 \\
\hline MUJERES MADURAS (41 - 60 años) & 0 & 0 & 1 \\
\hline VARONES ADULTOS ( 21 - 40 años) & 16 & 2 & 2 \\
\hline MUJERES ADULTAS ( 21 - 40 años) & 7 & 9 & 3 \\
\hline \hline ALOFISIOS ADULTOS & 2 & 0 & 0 \\
\hline ALOFISIOS INFANTILES II ( 7 - 12 años) & 5 & 6 & 0 \\
\hline & & & \\
\hline ALOFISIOS INFANTILES I ( 0-7 años) & 4 & 1 & 0 \\
\hline ALOFISIOS INFANTILES (no determinables) & 1 & 1 & 0 \\
\hline \hline N ${ }^{\circ}$ Total Individuos por tumba & 36 & 19 & 6 \\
\hline
\end{tabular}

Tab. 1. Tabla con la distribución de inhumados por tumbas, según sexo y edad del poblado calcolítico "Venta del Rapa" (Mancha Real, Jaén).

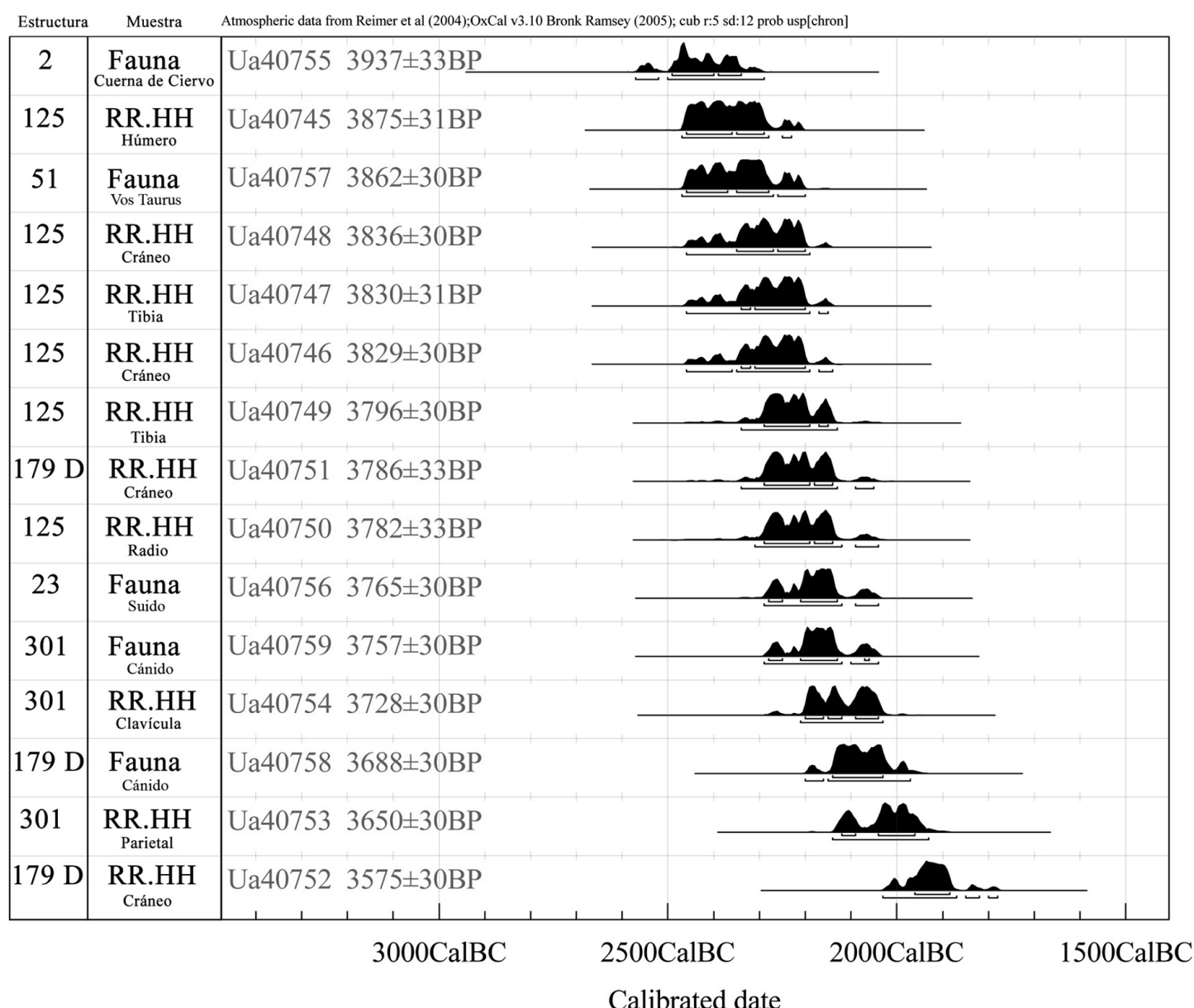

Fig. 8. Serie de gráficos de probabilidad de las calibraciones de dataciones C 14 de muestras procedentes del poblado calcolítico "Venta del Rapa" (Mancha Real, Jaén). RR HH Restos Humanos. 
nociendo el valor de este tipo de actividad económica en el seno de las comunidades calcolíticas del último tercio del III milenio a.C. Su inclusión en el sepulcro podría valorarse como un auténtico bien de prestigio.

La horquilla cronológica que arrojan las dataciones absolutas de las estructuras funerarias podría avalar la propuesta de que hubiera existido muchas más que albergarían los restos de la mayor parte de la población.

\section{LAS DATACIONES RADIOCARBÓNICAS}

Gracias a la colaboración con el Proyecto de Investigación (HAR2008-04577), “Cronología de la consolidación del sedentarismo y la desigualdad social en el Alto Guadalquivir", dirigido por J. A. Cámara, hemos datado por AMS 15 muestras de restos óseos humanos y faunísticos. El análisis fue realizado en el Laboratorio de la Uni- versidad de Uppsala (Suecia). La calibración se ha hecho a partir de la curva de P. J. Reimer (Reimer et al.2004) y del programa Oxcal 3.10 (Bronk Ramsey 2001) (Fig. 8).

La ocupación del poblado, según los materiales documentados y las muestras datadas, se encuadraría en el Calcolítico Pleno-Final (Fig. 9). La fecha más antigua disponible para el poblado, procede de la Estructura 2, localizada en el foso: Ua40755: $3937 \pm 33$ BP (2570-2290 2 $\sigma$ Cal. BC). Nos sitúa en la transición entre el Calcolítico Pleno y el Calcolítico Final ya en la ZAMB 2 (2450-2125 Cal. BC) propuesta para la cercana macro aldea de Marroquíes Bajos (Zafra et al. 2003). Todas las muestras de "Venta del Rapa" datadas se corresponden con las fases ZAMB 2 (2450-2125 Cal. BC) y ZAMB 3 (2125-1975 Cal. BC), mostrando la coetaneidad de esas fases de ocupación en ambos poblados (Zafra 2007). La muestra Ua40752 fecha el cese de la actividad y el abandono del poblado en $3575 \pm 30$ BP (1960$18852 \sigma \mathrm{Cal}$. BC). Es decir, según los materiales

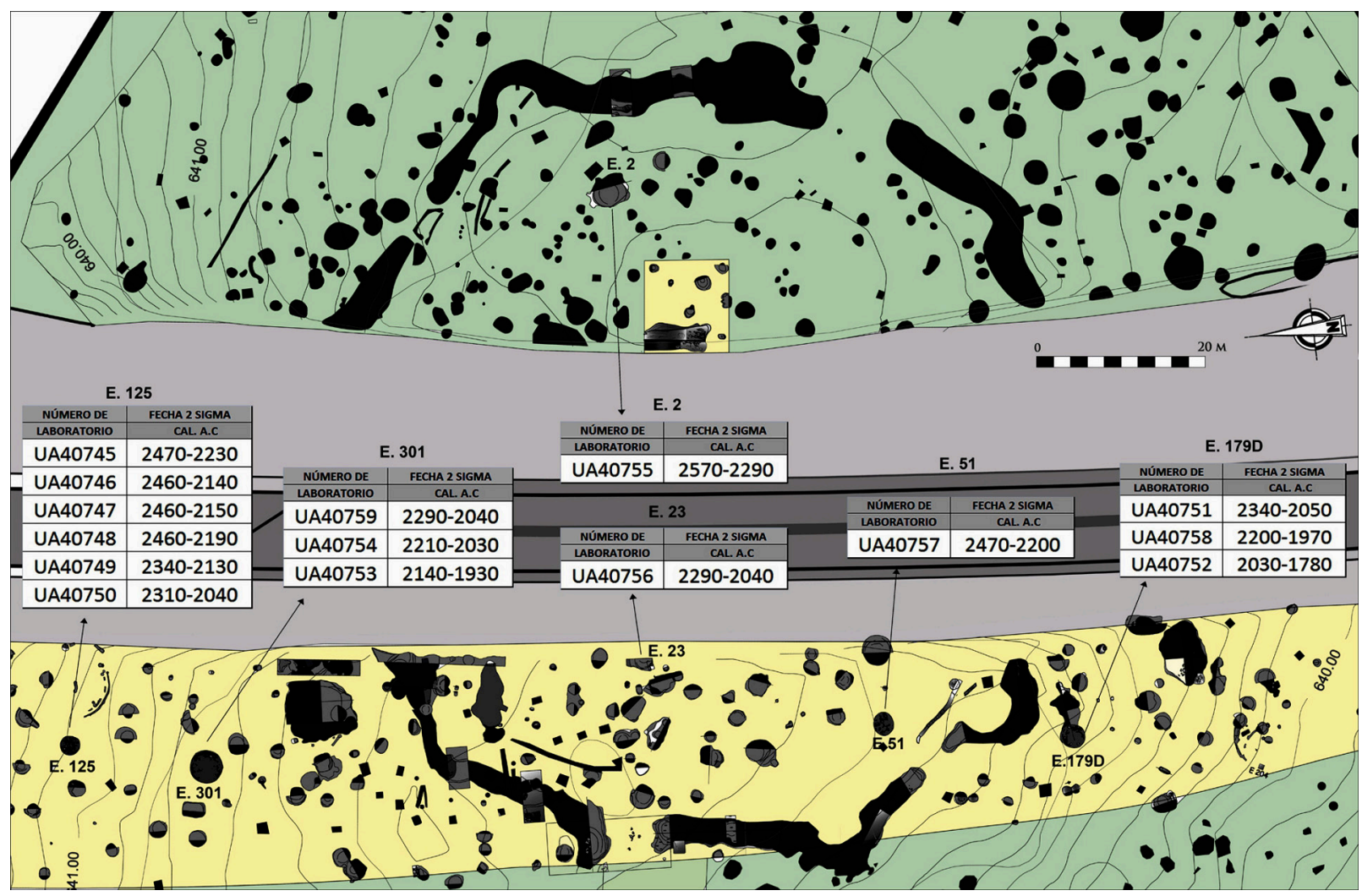

Fig. 9. Planta general del poblado calcolítico "Venta del Rapa" (Mancha Real, Jaén) con la localización de las muestras datadas.

Trab. prehist., 71, N. ${ }^{\circ}$ 2, julio-diciembre 2014, pp. 353-367, ISSN: 0082-5638 doi: $10.3989 /$ tp.2014.12139 
y las dataciones, la fase más antigua del poblado arranca con toda seguridad en torno al 2400-2350 Cal. BC. El período de máxima actividad dura entre el 2300 y $2100 \mathrm{Cal}$. BC, desapareciendo en torno al 2000-1950 Cal. BC. El poblado se ocupa durante 300-350 años.

Una vez fijada la escala temporal global, las dataciones nos permiten ahondar en la intrahistoria y observar el desarrollo de algunos de los acontecimientos más relevantes del poblado. La muestra con fecha más antigua, Ua40755: 3937 \pm 33 BP (2570-2290 2 $\sigma$ Cal. BC), procede de restos de cuernas, que procedían del estrato de abandono (US2) de una estructura de hábitat. Una muestra obtenida del húmero izquierdo de una mujer adulta fecha el inicio de las inhumaciones en la Estructura Funeraria 125, Ua40745, $3875 \pm$ 31 BP (2470-2230 2 $\sigma$ Cal. BC). La datación es coetánea a la del ritual de la E51, Ua40757: 3862 \pm 30 BP (2470-2200 2 $\sigma$ Cal. BC), durante el cual se depositó en la base de la tumba un bóvido casi completo, en conexión anatómica, así como restos de suidos y cérvidos. Son las estructuras más antiguas datadas que, posiblemente, inauguran y legitiman el poblado y probablemente la construcción del foso. El uso de la E125 como tumba colectiva perdura varias generaciones, al menos 80-100 años. Al segundo nivel de inhumaciones pertenecen las muestras Ua40748: $3836 \pm 30 \mathrm{BP}$ (2460-2190 2 $\sigma$ Cal. BC) y Ua40747: $3830 \pm 31$

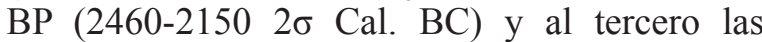
Ua40746: $3829 \pm 30$ BP (2460-2140 2 $\sigma$ Cal. BC); Ua40749: $3796 \pm 30$ BP (2340-2130 2 $\sigma$ Cal. BC)

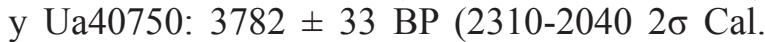
$\mathrm{BC})$.

Al clausurarse la tumba colectiva E125 se inaugura la E179D, Ua40751: $3786 \pm 33 \mathrm{BP}$

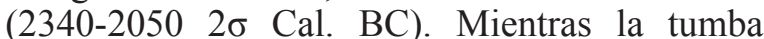
E179D está en uso, se están desarrollando actividades en el hábitat, en la E23, Ua40759: $3757 \pm$

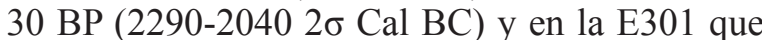
poco después se empieza a utilizar como tumba, Ua40754: $3728 \pm 30$ BP (2210-2030 2 $\sigma$ Cal. BC).

Según las muestras datadas, E301 y 179D conviven como tumbas colectivas donde se siguen realizando inhumaciones durante los últimos momentos de ocupación del poblado. La fecha más reciente, obtenida en la tumba E179D, Ua40753: $3575 \pm 30$ BP (2140-1930 2 $\sigma$ Cal. BC), sitúa el fin del poblado en el tránsito entre el III y el II milenio a.C.

\section{VALORACIÓN GLOBAL}

La planificación y ejecución de una serie de medidas correctoras en las obras de la autovía del Olivar (A-316) ha permitido una gestión integral del poblado calcolítico "Venta del Rapa". Su destrucción parcial, en la ejecución de la antigua carretera, no ha impedido documentar la extensión casi total del mismo. Cientos de estructuras excavadas en la base geológica que fueron protegidas y soterradas tras la modificación puntual de ciertas actuaciones proyectadas para la construcción de la autovía. Solo se excavó lo que, finalmente, iba a ser afectado por los desmontes del tronco de la autovía.

Somos conscientes que es complicado establecer un uso coetáneo de las centenares de estructuras durante toda la ocupación del poblado, sin embargo hemos identificado diferencias morfológicas y constructivas en las estructuras de 'hábitat', que podrían indicar su evolución temporal. El foso o zanja sobresale como estructura más emblemática del poblado. Con una morfología de tendencia circular de unos $70 \mathrm{~m}$ de diámetro delimitaba un espacio de casi 0,5 ha. Es similar al Foso 0 de Marroquíes Bajos (Rodríguez Ariza et al. 2005: 587, 2006: 286), aunque con una cronología más reciente. Su construcción exige el esfuerzo conjunto de la pequeña comunidad que lo construye, producto de un proceso de agregación de varios grupos familiares. De este modo, el foso estaría actuando como medio de afirmación de la cohesión interna de la comunidad. Carecemos de muestras fechadas de su uso, pero podemos situarlo entre 2350-2000 Cal. BC. En este momento ya no se fundan asentamientos con fosos y solo algunos de los mayores de la etapa precedente experimentan una actividad constructiva (Márquez y Jiménez 2010: 206)

En la base económica y subsistencial del poblado, la ganadería jugó un papel muy por encima de la incipiente agricultura, con un predominio del ganado porcino y vacuno sobre el ovicáprino. La importancia de la ganadería pudo favorecer desplazamientos estacionales de parte de la comunidad en busca de pastos por un territorio más o menos restringido. La ausencia casi total de elementos alóctonos (molinos de granito, cobre...) nos puede indicar la situación periférica de esta comunidad con respecto a los 'circuitos comerciales' de la campiña del Guadalquivir o del valle del Guadalbullón, así como la ausencia 
de una 'élite social' que demandara estos productos. La existencia de, al menos, una estructura con una deposición intencional de restos de fauna, nos plantea la práctica de rituales en el poblado (Cámara et al. 2008:79), destinados, tal vez, a cohesionar el grupo, legitimar el poblado y afirmar la comunidad.

El rito funerario de la comunidad carece de elementos de ajuar en las tres tumbas colectivas documentadas, en uso durante varias generaciones. Ello puede estar reflejando unidades familiares extensas (¿clanes?). Tampoco hay indicadores de un inicio de desigualdad o jerarquización aunque debemos destacar la presencia de canes con los inhumados en dos tumbas. Planteamos que el sistema de relaciones sociales del grupo humano que habitó 'Venta del Rapa' no era estratificado, ni tan siquiera jerarquizado (excluyendo lo que pueda derivarse por los estatus de edad y género). Se basaba exclusivamente en el parentesco, donde lo comunal y lo colectivo tienen una importante función como soporte del esquema de relaciones sociales. El grado de cohesión y compacidad internas de las unidades familiares es tan alto que la ideología predominante impone que los individuos de una generación sean enterrados en las mismas estructuras funerarias (probablemente ya osarios en realidad) que los de las generaciones anteriores. En la esfera de las relaciones sociales de producción, este grupo humano de las etapas finales de la Edad del Cobre asume un esquema basado exclusivamente en el parentesco y de base comunal en el que el producto circula mediante mecanismos de redistribución, sin apropiación diferencial por parte de un segmento de la comunidad.

\section{BIBLIOGRAFÍA}

Arribas, A. y Molina, F. 1979: El poblado de 'Los Castillejos' en la Peña de los Gitanos Montefrío, Granada). Campaña de excavaciones de 1971. El Corte núm. 1. Cuadernos de Prehistoria de la Universidad de Granada, Serie Monográfica 3. Granada.

Blasco, C. y Ríos, P. 2012: "La mujer en el III milenio a.C. a través de las manifestaciones funerarias: un ejemplo en territorio madrileño". En L. Prados (ed.): La arqueología funeraria desde una perspectiva de género. Universidad Autónoma de Madrid. Madrid: 39-54.
Bronk Ramsey, C. 2001: "Development of the radiocarbon calibration program OxCal". Radiocarbon 43 (2A): 353-363.

Cámalich, M. D. y Martín, D. 1999: El territorio almeriense desde los inicios de la producción hasta fines de la antigüedad. Un modelo: la depresión de Vera y cuenca del Río Almanzora. Arqueología, Monografías, Consejería de Cultura, Junta de Andalucía, Sevilla.

Cámara, J. A. 2001: El ritual funerario en la prehistoria reciente en el sur de la Península Ibérica. British Archaeological Reports, International Series 913. Archaeopress. Oxford: 1-346.

Cámara Serrano, J. A.; Lizcano Prestel, R.; Pérez Bareas, C. y Gómez Del Toro, E. 2008: "Apropiación, sacrificio, consumo y exhibición ritual de los animales en el Polideportivo de Martos. Sus implicaciones en los orígenes de la desigualdad social". Cuadernos de Prehistoria y Arqueología de la Universidad de Granada 18: 55-90.

Díaz-del-Río, P. 2003: "Recintos de fosos del III Milenio AC en la Meseta Peninsular". Trabajos de Prehistoria 60 (2): 61-78.

Fernández, M. 2008: "Análisis tipológico y tecnológico de los conjuntos cerámicos de la Motilla del Azuer (Daimiel, Ciudad Real)". Cuadernos de Prehistoria y Arqueología de la Universidad de Granada 18: 317-356.

Harris, E. 1991: Principios de la Estratigrafía Arqueológica. Crítica. Barcelona.

Lizcano Prestel, R. 1999: El polideportivo de Martos (Jaén): un yacimiento neolítico del IV Milenio AC. Nuevos datos para la reconstrucción del Proceso Histórico del Alto Guadalquivir. Cajasur. Córdoba.

Lomba Maurandi, J.; Lopez Martínez, M.; Ramos Martínez, F. y Avilés Fernández, A. 2009: "El enterramiento múltiple calcolítico de Camino del Molino (Caravaca, Murcia). Metodología y primeros resultados de un yacimiento excepcional". Trabajos de Prehistoria 66 (2): 143-159.

Márquez Romero, J. E. y Jiménez Jáimez, V. 2010: Recintos de fosos. Genealogía y significado de una tradición en la Prehistoria del suroeste de la Península Ibérica (IV-III milenios $A C$ ). Universidad de Málaga. Málaga.

Murillo Redondo, J. F.; Ruiz López, A.; Ruiz Gómez, A. M. ${ }^{\text {a }}$ y Araque Aranda, F. A. 1991: "Materiales calcolítico procedentes de 'La Longuera' (El Viso, Córdoba)". Anales de Arqueología Cordobesa 2: 53-98.

Otero, J. 1985: "Estudio tipológico de las formas cerámicas llamadas 'platos' en el Calcolítico de Andalucía”. Habis 17: 403-432.

Reimer, P. J.; Baillie, M. G. L.; Bard, E.; Bayliss, A.; Beck, J. W.; Bertrand, C. J. H.; Blackwell, P. G.; Buck, C. E.; Burr, G. S.; Cutler, K. B.; Damon, P. E.; Edwards, R. L.; Fairbanks, R. G.; Friedrich,

Trab. prehist., 71, N. ${ }^{\circ}$ 2, julio-diciembre 2014, pp. 353-367, ISSN: 0082-5638

doi: $10.3989 /$ tp.2014.12139 
M.; Guilderson, T. P.; Hogg, A. G.; Hughen, K. A.; Kromer, B.; Mccormac, G.; Manning, S.; Bronk Ramsey, C.; Reimer, R. W.; Remmele, S.; Southon, J. R.; Stuiver, M.; Talamo, S.; Taylor, F. W.; Van Der Plicht, J. y Weyhenmeyer, C. E. 2004: "IntCal04 Terrestrial radiocarbon age calibration, 0-26 calkyr BP". Radiocarbon 46, 3: 1029-1058.

Rodríguez-Ariza, M. O.; Luna, M. B.; Montes, E. y Visedo, A. 2005: "Intervención arqueológica realizada en la Parcela $C$ del sector urbanístico Residencial Programado n. 4 (RP4) de Marroquíes Bajos (Jaén) (Campaña de 2002)". Anuario Arqueológico de Andalucía 2002, III-1. Junta de Andalucía. Sevilla: 583-592.

Rodríguez-Ariza, M. O.; Luna, M. B.; Montes, E. y Visedo, A. 2006: "II Campaña de excavación en la Parcela C de Marroquíes Bajos (Jaén)". Anuario Arqueológico de Andalucía 2003. II-1. Junta de Andalucía. Sevilla: 281-290.
Serrano Peña, J. L.; Portero Fernández, V. y Cano Carrillo, J. 2011: Marroquies Bajos en su tiempo y arqueología urbana en Jaén. Intervención arqueológica en el Centro Comercial El Corte Inglés de Jaén. El Corte Inglés, Ámbito Cultural. Granada.

Zafra de la Torre, N. 2007: De los campamentos nómadas a las aldeas campesinas. La provincia de Jaén en la Prehistoria. Universidad de Jaén. Jaén.

Zafra de la Torre, N.; Castro, M. y Hornos, F. 1999: "Una macro-aldea en el origen del modo de vida campesino: Marroquíes Bajos (Jaén) c. 2500-2000 cal. A.N.E.". Trabajos de Prehistoria 56 (1): 77102.

Zafra de la Torre, N.; Castro, M. y Hornos, F. 2003: "Sucesión y simultaneidad en un gran asentamiento: la cronología de la macro-aldea de Marroquíes Bajos (Jaén) c. 2500-2000 cal. A.N.E.”. Trabajos de Prehistoria 60 (2): 79-90. 